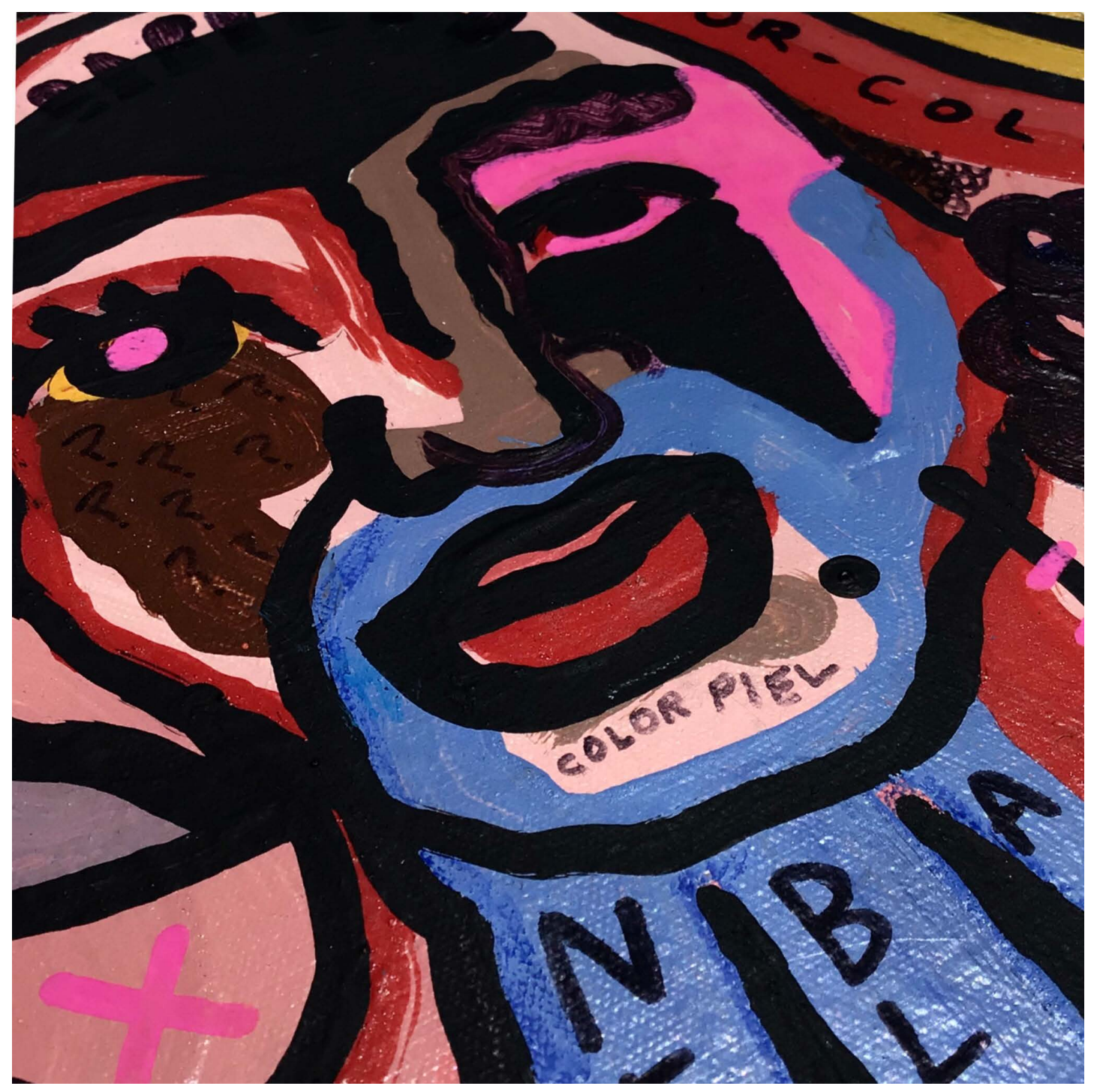

Artista invitado

Fabio Andrés Arboleda Mendoza

Color piel (detalle)

Acrílico sobre lienzo

$30 \times 35 \mathrm{~cm}$

2016

Medellín 


\title{
Explicar los impactos de la globalización en las zonas transfronterizas a través de la calidad de la democracia. Un marco interregional comparativo*
}

\author{
Harlan Koff (Estados Unidos) ${ }^{* *}$ \\ Santiago Mejía Idárraga (Colombia) $)^{* * *}$
}

\section{Resumen}

La globalización afecta significativamente la capacidad de los Estados para gobernar los flujos. Dos preguntas importantes surgen recientemente en las relaciones internacionales: ¿cómo ha afectado la globalización a las zonas fronterizas? y ¿qué explica estos impactos? A partir de la implementación de un diseño de investigación interregional comparativo, este artículo propone dos originalidades como respuesta: primero, cuestiona si las comunidades fronterizas tienden a beneficiarse o a sufrir la globalización, y aborda a su vez los impactos de la globalización en las comunidades fronterizas; en segundo lugar, se intenta explicar estos impactos mediante el estudio

[294] de la calidad democrática de los procesos de integración fronteriza. Se examina así la globalización como un sistema en el que los beneficios se distribuyen o concentran en función de la calidad de los procesos de integración.

\section{Palabras clave}

Relaciones Internacionales; Fronteras; Globalización; Integración Regional; Método Comparativo; Calidad de la Democracia.

Fecha de recepción: noviembre de 2020 - Fecha de aprobación: junio de 2021

\footnotetext{
* Investigación financiada por la Universidad de Luxemburgo a través del proyecto Human and Environmental Security in Cross-Border Regions (HUMENITY) y por el Consortium for Comparative Research of Regional Integration and Social Cohesion-Social Elevation (RISC-RISE), a través de una beca de intercambios científicos.

** Politólogo. Magíster y doctor en Ciencia Política. Profesor de Ciencias Sociales, Universidad de Luxemburgo. Cátedra GAMMA-UL de Integración Regional y Sustentabilidad en el Instituto de Ecología, AC (INECOL) en México. Investigador Asociado Senior, Departamento de Política y Relaciones Internacionales, Universidad de Johannesburgo, Sudáfrica. Correo electrónico: harlan. koff@uni.lu - Orcid: 0000-0002-9799-0893 - Google Scholar: https://scholar.google.com/citations?us er=ARBKxDoAAAAJ\&hl=es

**** Politólogo. Magíster en Hábitat. Doctor en Ciencias Sociales. Docente de la Universidad de Medellín, Colombia. Correo electrónico: samejia@udem.edu.co - Orcid: 0000-0001-6417-4550
} 


\title{
Cómo citar este artículo
}

Koff, Harlan y Mejía Idárraga, Santiago. (2021). Explicar los impactos de la globalización en las zonas transfronterizas a través de la calidad de la democracia. Un marco interregional comparativo. Estudios Políticos (Universidad de Antioquia), 62, pp. 294-321. https://doi.org/10.17533/udea.espo.n62a12

\section{Explaining the Impacts of Globalization on Borderlands through Quality of Democracy. A Comparative Cross- Regional Framework}

\begin{abstract}
Globalisation has significantly affected the ability of States to govern flows. Important questions which have recently emerged in international relations ask: how has globalisation affected border regions and what explains these impacts? Employing a comparative, cross-regional research design, this article proposes two originalresponses to these questions: first, it questions whether border communities benefit or suffer from globalization and, in doing so, it addresses the impacts of globalization on border communities. Second, an attempt is made to explain these impacts by studying the democratic quality of border integration processes. Thus, globalization is examined as a system in which the benefits are distributed or concentrated according to the quality of the integration processes.
\end{abstract}

\section{Keywords}

International Relations; Borders; Globalisation; Regional Integration; Comparative Method; Quality of Democracy. 


\section{Introducción}

La globalización, definida como el establecimiento de sistemas económicos, políticos, sociales y de comunicaciones globales (Vivekanandan, 2021) afecta significativamente la capacidad de los Estados para gobernar los flujos de población, capital e información. En este contexto, dos preguntas importantes surgen recientemente en las relaciones internacionales: ¿cómo ha afectado la globalización a las zonas fronterizas? y ¿qué explica estos impactos?

Las zonas fronterizas se destacan en los debates sobre la globalización en relación con el surgimiento de flujos y redes transnacionales, ya que la literatura a menudo sostiene que la globalización disminuye la relevancia de las fronteras estatales y aumenta el valor estratégico de las zonas transfronterizas (Koff, 2015). Si bien esta literatura es rica, la construcción de teorías se ve obstaculizada por el análisis de contextos o casos específicos. En consecuencia, en respuesta a las preguntas de investigación, este artículo propone dos originalidades: en primer lugar, presenta una investigación comparativa interregional dirigida a la construcción de teorías más que a una explicación específica de casos en una región, empleando tipologías

y escalas para armonizar el análisis de diferentes casos de investigación —véase más adelante una explicación más detallada-; en segundo lugar, identifica la «calidad de la democracia» como fundamental para comprender la globalización en las zonas transfronterizas.

\section{Diseño de la investigación y métodos}

La literatura acerca de zonas fronterizas se caracteriza por estudios sobre contextos específicos (Ceballos, 2011; Hu y Konrad, 2021; Sandoval, 2017). Dado que este artículo se centra más en vincular enfoques teóricos que en la explicación de casos específicos, implementa un diseño de investigación interregional comparativo. La investigación presenta un estudio empírico sobre siete zonas transfronterizas: i) la Eurometrópolis franco-belga; ii) la Gran Región de Luxemburgo; iii) Bari, Italia-Durrës, Albania; iv) Melilla, España-Nador, Marruecos; v) San Diego, Estados Unidos-Tijuana, México; vi) Chetumal, México-Corozal, Belice; y vii) Cúcuta, Colombia-San Cristóbal, Venezuela (véase mapa 1). 


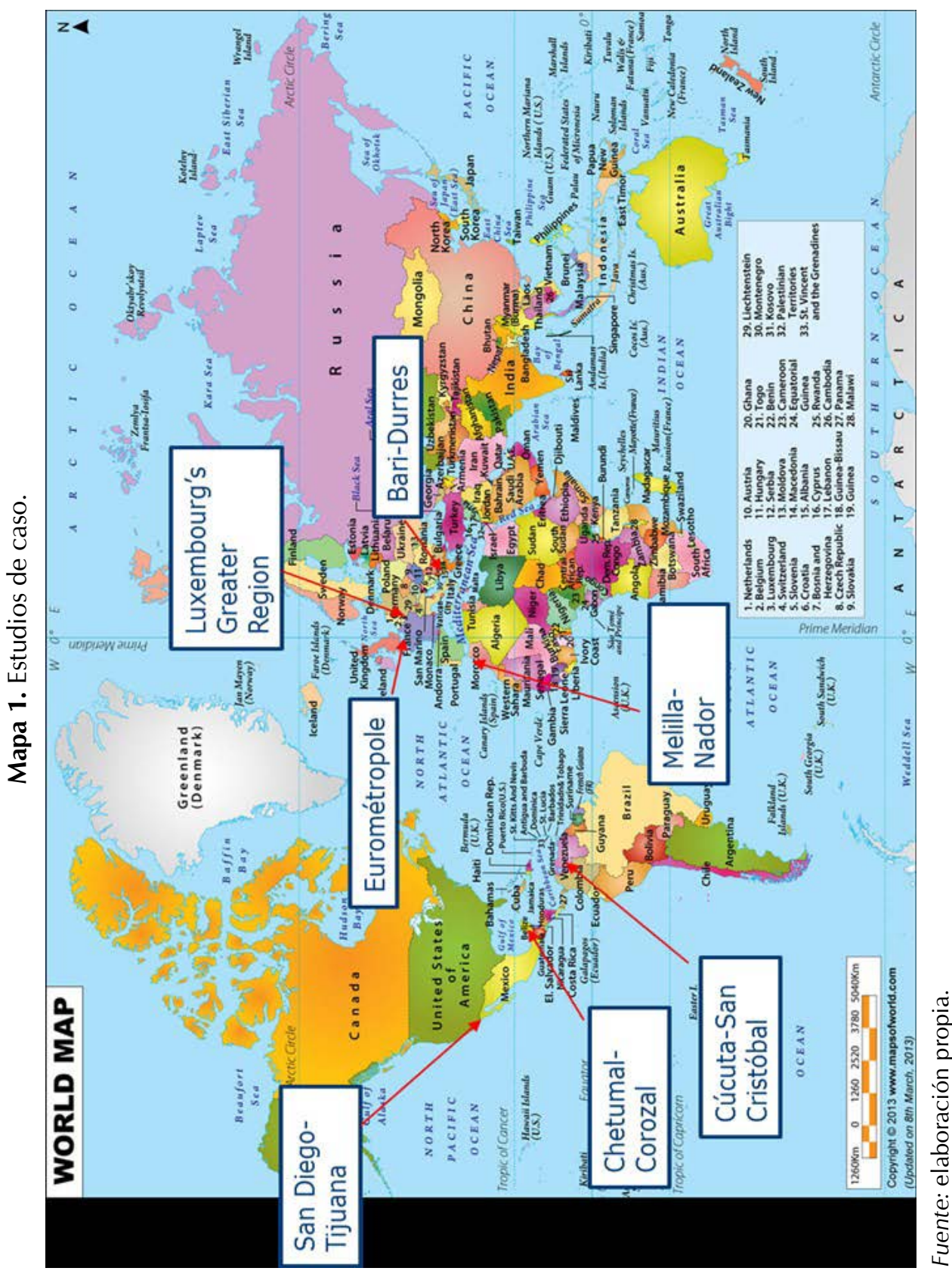

[297] 
Estos casos fueron elegidos para incluir dos fronteras internas de la Unión Europea (UE), dos fronteras externas de la UE, una frontera interna del Tratado de Libre Comercio de América del Norte (TLCAN)-Tratado entre México, Estados Unidos y Canadá (T-MEC), una frontera externa del TLCAN/ T-MEC y una frontera de la Comunidad Andina (CAN). La comunidad Cúcuta-San Cristóbal representa dos casos, porque Venezuela se retiró de la CAN en 2006, por lo tanto, el proyecto estudia el área como frontera interna y externa de la CAN, e incluye a su vez ocho casos en siete comunidades.

El diseño de investigación comparativa interregional se guía por los enfoques que caracterizan la literatura sobre la globalización (cuadro 1) a través del análisis de agrupaciones basadas en contextos de gobernanza regional -globalización como proceso-, niveles de integración económica transfronteriza — globalización como ideología- o la presencia-ausencia de movilización transfronteriza —globalización impulsada por actores-.

Cuadro 1. Investigación comparativa interregional para pruebas de hipótesis.

\begin{tabular}{|c|c|c|}
\hline Globalización como proceso & $\begin{array}{l}\text { Globalización } \\
\text { como ideología }\end{array}$ & $\begin{array}{c}\text { Globalización impulsada } \\
\text { por actores }\end{array}$ \\
\hline $\begin{array}{l}\text { - Agrupación 1: frontera } \\
\text { interna de la UE } \\
\text { - Gran Región de Luxemburgo. } \\
\text { - Eurometrópolis. } \\
\text { - Agrupación 2: frontera } \\
\text { externa de la UE } \\
\text { - } \text { Melilla-Nador. } \\
\text { - } \text { Bari-Durrës. } \\
\text { - Sin agrupación } \\
\text { - San Diego-Tijuana (Frontera } \\
\text { interna del TLCAN/T-MEC). } \\
\text { - Chetumal-Corozal (Frontera } \\
\text { externa del TLCAN/T-MEC). } \\
\text { - Cúcuta-San Cristóbal (Frontera } \\
\text { interna y externa de la CAN). }\end{array}$ & $\begin{array}{l}\text { - Agrupación 2: mercados } \\
\text { globalizados } \\
\text { - San Diego-Tijuana. } \\
\text { - Gran Región de } \\
\text { - Luxemburgo. } \\
\text { - Agrupación 2: mercados } \\
\text { globalizados } \\
\text { - Bari-Durrës. } \\
\text { - Eurometrópolis. } \\
\text { - Agrupación 3: mercados } \\
\text { localizados } \\
\text { - Melilla-Nador. } \\
\text { - Chetumal-Corozal. } \\
\text { - Cúcuta-San Cristóbal. }\end{array}$ & $\begin{array}{l}\text { - Agrupación 1: } \\
\text { movilización } \\
\text { transfronteriza } \\
\text { - San Diego-Tijuana. } \\
\text { - } \text { Cúcuta-San Cristóbal. } \\
\text { - } \text { Bari-Durrës. } \\
\text { - Agrupación 2: } \\
\text { movilización } \\
\text { transfronteriza limitada } \\
\text { - } \text { Gran Región de } \\
\text { Luxemburgo. } \\
\text { - Eurometrópolis. } \\
\text { - } \text { Melilla-Nador. } \\
\text { - Chetumal-Corozal. }\end{array}$ \\
\hline
\end{tabular}

Fuente: elaboración propia.

El problema para los estudios interregionales es la falta de datos comparables, porque bases de datos transfronterizos no existen en esta 
escala. Para hacer operativa la información y hacerla comparable, este artículo se basa en la investigación cualitativa implementada en cada caso de estudio. Al utilizar las metodologías presentadas en los índices de globalización - como el índice de globalización KOF, el índice de globalización de Maastricht, entre otros-, el artículo establece escalas de impacto de la globalización. Estas escalas incluyen categorías temáticas derivadas de los índices antes mencionados.

Para «medir» impactos de la globalización, el análisis crea indicadores basados en el trabajo de Jon Elster (1992), Local Justice, en el que se sostiene que el bienestar de los individuos depende de su éxito en la obtención de bienes y de evitar las cargas distribuidas por la sociedad. Para hacer operativo este enfoque, Elster estudia empíricamente diferentes temas relacionados con la justicia social y emplea etiquetas de «» y «+» para representar «Costos» $y$ «beneficios». Si bien este enfoque simplifica la «justicia», propone una lógica universal para comprender la relación entre globalización y desarrollo regional en diferentes contextos políticos, económicos y sociales transfronterizos.

Al seguir la lógica empleada por Elster (1992), esta investigación presenta escalas ordinales que indican niveles e impactos de la globalización relacionados con costos y beneficios:

1 = beneficios positivos globalizados y generalizados para la zona transfronteriza.

0,5 = beneficios positivos globalizados para la zona transfronteriza, pero concentrados en una parte de la frontera.

$0=\sin$ impactos significativos.

$-0,5=$ costos negativos globalizados para la zona transfronteriza y concentrados en una parte de la frontera.

$-1=$ costos negativos globalizados y generalizados para la zona transfronteriza.

Se calculan las puntuaciones generales del impacto de la globalización y luego en el análisis se observa si surgen las agrupaciones antes mencionadas.

Para explicar los impactos de la globalización en zonas fronterizas se presentan escalas nominales como un medio para calcular las puntuaciones 
de "calidad transfronteriza de la democracia». Se toman en cuenta las cualidades positivas y negativas existentes en los casos de investigación, así como las dimensiones formales e informales de cada categoría. La escala construida a partir de la interacción de estos factores es la siguiente:

1 = calidad neta formal e informal positiva transfronteriza.

$0,5=$ calidad neta formal o informal positiva transfronteriza.

$0=$ calidad neta ni positiva ni negativa transfronteriza.

$-0,5=$ calidad neta formal o informal negativa transfronteriza.

$-1=$ calidad neta formal e informal negativa transfronteriza.

El artículo compara estos puntajes generales sobre la calidad de la democracia con los puntajes de impacto de la globalización.

\subsection{Recolección de datos}

Los datos se recopilan de revisiones de literatura, documentos de la UE, la Organización de Estados Americanos (OEA) y documentos de gobiernos nacionales, informes de organizaciones no gubernamentales (ONG) y bases de datos de la ONU, nacionales y locales. Se conducen entrevistas seleccionadas con representantes de gobiernos locales, ONG y académicos a través de varias visitas de investigación entre 2000 y 2016. Este extenso periodo de tiempo facilita el examen de la evolución del desarrollo en las comunidades fronterizas.

\section{Integración de las globalizaciones y las fronteras: imbricación de dos literaturas}

La globalización se define y utiliza de tantas formas y contextos que David Held, Anthony McGrew, David Goldblatt y Jonathan Perraton (1999) se refieren a la «globalización» como el cliché de nuestro tiempo. Jan Aart Scholte (2004) sostiene que este fenómeno debería abordarse como "globalizaciones», dada la presencia de procesos o sistemas en competencia, pero interrelacionados. Se proponen innumerables enfoques para el estudio de las globalizaciones, sin embargo, estos estudios generalmente pueden organizarse en tres subgrupos: a) la globalización como un proceso; b) la globalización como una ideología; y c) la globalización como sistemas impulsados por actores. 


\subsection{Globalización como proceso}

La globalización se entrelaza con las nociones de interconexión. Algunos académicos discuten la relación entre tiempo y espacio en la que se aceleran las interacciones humanas. Gary Gereffi (1994) discute las relaciones económicas entre sociedades, reconoce el control global de la producción a través de «cadenas de productos básicos» y aborda la capacidad de las comunidades nacionales para trabajar juntas en la arena global.

Estas aproximaciones a la globalización, de hecho, abordan el declive del Estado-nación como la unidad de gobierno más eficiente para la regulación económica. Académicos como Ronald Rogowski (1990) identifican cambios de poder en los sistemas capitalistas desde que supuestamente comienza la globalización en la década de 1980. Mientras que la política en los Estados industriales avanzados se organiza en el siglo xx en torno a divisiones de clases y la regulación estatal territorial, el siglo xxı es testigo de coaliciones y divisiones políticas desterritorializadas, lo que da lugar a discusiones sobre la gobernanza económica en términos de flujos (Dubé, 2020).

Estos niveles crecientes de interconexión globalizada llevan a una investigación teórica y empírica que aborda subpreguntas importantes, la más destacada la formula Mauro F. Guillén (2001): ¿ocurre realmente la globalización? En respuesta, Risto Tainio, Susan Meriläinen, Jukka Mäkinen y Maarit Laihonen (2014) examinan el comercio mundial y las conexiones empresariales con el fin de indicar empíricamente si se produce un cambio real en los sistemas económicos. De manera similar, Manuel Castells (2003) discute la «Era de la Información», relacionada con la globalización de la información y la tecnología, examinada empíricamente por académicos como Michalinos Zembylas y Charalambos Vrasidas (2005). Otros (Fligstein y Habinek, 2014) investigan la proliferación y velocidad de las conexiones financieras.

Otro aspecto de esta investigación se centra en las consecuencias de la globalización. Axel Dreher y Noel Gaston (2006), entre otros, investigan el impacto de la globalización en la desigualdad. Pim Martens y Mohsin Raza (2010) estudian la relación entre globalización y sostenibilidad. Esta rama de la investigación en estudios de globalización también promueve numerosos índices que intentan medir la globalización y sus impactos, por ejemplo: el KOF Globalization Index (ETH Zürich KOF, s. f.) y Maastricht Globalization 
Index (Pim Martens.info, s. f.) proporcionan datos nacionales que «miden» los niveles de globalización en los ámbitos político, económico, social y cultural. El propósito de estos índices es demostrar cuánta globalización se produce entre casos; sin embargo, la interpretación de estos datos y una comprensión general del significado de globalización en realidad se relacionan más con un subcampo diferente.

\subsection{Globalización como ideología}

De hecho, no todos los investigadores ven la globalización de manera objetiva. Otro subcampo de los estudios de globalización examina la globalización como ideología. Según este enfoque, la globalización está intrínsecamente ligada a la democracia liberal y al capitalismo. Así, la globalización representa más que un proceso, es una ideología que presenta valores fundamentales. Las preguntas más importantes que plantea esta literatura son: ¿la globalización promueve la convergencia social? ¿Está surgiendo una cultura global? Si es así, ¿qué es?

Los académicos que utilizan este enfoque destacan la importancia del significado. Ino Rossi (2007) analiza la construcción social del espacio y el

[302 ] lugar en relación con la globalización, y describe el «lugar» como un «espacio» construido socialmente en el que se atribuye un significado subjetivo a los procesos supranacionales. Debido a que estos procesos trascienden los Estados-nación, la globalización a menudo se ve como una homogeneización cultural que socava las identidades nacionales y promueve una conciencia global singular. En algunos casos, los académicos analizan los impactos de la globalización económica en los valores políticos. Por ejemplo, Axel Dreher, Martin Gassebner y Lars Siemers (2012) estudian el impacto de las conexiones económicas globalizadas en los compromisos nacionales con los derechos humanos. Otros estudiosos analizan la globalización como una ideología bajo la cual proliferan los valores políticos globalizados que ocultan las afiliaciones nacionales. Esto se materializa en la referencia a «McWorld» en la obra fundamental de Benjamin Barber (1995), Jihad vs. McWorld.

Estos valores se pueden difundir de diferentes formas. Los estudios sobre la Unión Europea en asuntos globales (Häbel, 2020) examinan la difusión de normas y el uso de la condicionalidad en las políticas exteriores y de desarrollo de la UE. Si bien estos estudios identifican diferentes 
mecanismos como claves para la homogeneización cultural o identitaria, su vínculo común es su enfoque en las identidades «globalizadas». La globalización se ve como una construcción que abarca valores fundamentales que trascienden los Estados-nación. Por supuesto, esto es relevante para las relaciones internacionales porque resalta la importancia de los actores no estatales en los asuntos globales. El surgimiento de estos actores es el foco de la tercera vertiente de la literatura sobre globalización.

\subsection{Globalización como sistemas impulsados por actores}

¿Cómo se promueve la globalización? Diversos académicos se preguntan si la globalización realmente representa el surgimiento de «un nuevo orden global» o si es simplemente el uso estratégico de nuevos niveles de gobernanza por parte de los actores políticos para perseguir agendas políticas. Esta visión de la globalización emerge en la literatura sobre "globalizaciones». Uno de los mayores defensores de este enfoque es Gustavo Lins Ribeiro (2009), que no niega que los procesos económicos internacionales y las hegemonías culturales globales surgen desde la década de 1990; sin embargo, sostiene que el surgimiento de hegemonías culturales y económicas es acompañado de globalizaciones no hegemónicas que responden a ellas. En concreto, identifica el establecimiento de movimientos sociales transnacionales mediante el uso de tecnologías de la comunicación como un medio para contrarrestar la globalización económica y la hegemonía cultural. Este enfoque también se adopta por académicos en otros campos, como la seguridad internacional, con autores como Sean Kay (2004), que argumentan que la globalización altera el uso del poder en los asuntos globales, Por estas razones, el campo de la globalización es relevante para los estudios fronterizos.

\subsection{Globalización y zonas fronterizas: una revisión crítica}

En 2010, La Vie-Le Monde publicó un número especial titulado L'Atlas des Mondialisations -El Atlas de las globalizaciones- (Denis y Greilsamer, 2010). Si bien no se centra específicamente en la integración fronteriza, este número incluye numerosos textos breves de observadores de la globalización reconocidos internacionalmente que abordan importantes cuestiones para nuestra comprensión de las zonas transfronterizas contemporáneas. El artículo principal del número especial marca el tono de este análisis: «Sociedad civil global, economía global: no existen. Múltiples redes 
globales se cruzan en todo el planeta, cada una distinta y caracterizada por fuertes particularidades, más o menos especializadas, que conectan partes específicas de las ciudades: esta es la principal característica del período contemporáneo» (Sassen, 2010, p. 10. Traducción propia). En resumen, este número especial conceptualiza la «globalización» como «transnacional», en contraposición a «internacional», y vincula el concepto a ciudades interconectadas.

Estos puntos de vista tienen significado para las zonas fronterizas. Desde el final de la Guerra Fría y la proliferación de la integración regional en la década de 1990, las fronteras reciben una atención significativa en los asuntos globales, supuestamente por su «desaparición». Al respecto, la literatura sobre el «mundo sin fronteras» (Ohmae, 1999) documenta el flujo transnacional de capitales, bienes y servicios. Esta tendencia en la economía, sin embargo, produce un efecto contrario en las políticas de seguridad, que lleva a los Estados a reforzar los controles fronterizos, a menudo mediante la construcción de muros y barreras (Koff, 2017).

La literatura en los estudios de las zonas fronterizas documenta esta dicotomía interactiva a través de diferentes enfoques disciplinarios que en

[304 ] realidad reflejan las ramas de la globalización presentadas anteriormente. En el campo de la economía política, académicos como James Gerber (2020) discuten la formación de sistemas económicos transfronterizos globalizados en los que las ciudades fronterizas colaboran con sus contrapartes en el lado opuesto de las divisiones nacionales más que con sus propias capitales nacionales, lo que disminuye así el peso de los modelos económicos nacionales. Este enfoque refleja el paradigma de la «globalización como proceso». Un tema significativo en este enfoque se centra en el establecimiento de metrópolis transfronterizas (Herzog y Sohn, 2014) y «áreas metropolitanas transfronterizas policéntricas» (De la Mora-De la Mora, 2017). Una parte de esta literatura incluso discute la evolución de las economías informales en las que las fronteras se ven como recursos para «estrategias de supervivencia» (Faleye, 2016).

El enfoque de la «globalización como ideología» se adopta en los estudios sobre las zonas fronterizas mediante el paradigma de la «construcción social de las fronteras». Este enfoque de los estudios sobre las zonas fronterizas resta importancia al papel de los gobiernos nacionales en la dinámica de las ciudades fronterizas al destacar la cooperación 
transfronteriza diaria de las poblaciones urbanas. Académicos activos en este campo, como Diana Morales (2020) y Elisabetha Nadalutti (2020) señalan que las comunidades locales en las ciudades fronterizas fomentan los lazos para promover identidades desterritorializadas, muchas de las cuales están profundamente arraigadas en relaciones históricas que son anteriores a las fronteras contemporáneas. La noción de «paisajes fronterizos» es particularmente relevante para este enfoque, ya que este paradigma destaca la interacción entre la construcción subjetiva de fronteras "posnacionales» y «posmodernas» y los procesos relacionados con la «fronterización» y la «desfronterización» (Liberona, Álvarez y Córdoba, 2018).

Finalmente, la literatura sobre fronteras y globalización documenta el reforzamiento de las fronteras en diferentes partes del mundo y las reacciones contra estas medidas entre las organizaciones transfronterizas. Esto refleja así el enfoque de «globalización como un sistema impulsado por actores». Autores como Low Choo Chin (2019) y Marc Brightman y Vanessa Grotti (2014) documentan la «securitización» ${ }^{1}$ de las fronteras desde el 11 de septiembre de 2001. Estos estudios discuten la «securitización» en detalle, en términos de narrativas políticas, imperativos de políticas, mecanismos de implementación y tecnología. Por el contrario, estudiosos como Richard Meissner y Jeroen Warner (2021) y Dudziro Nhengu (2020) documentan la proliferación de movimientos sociales transfronterizos que se oponen a estas medidas, que reflejan así el trabajo sobre globalizaciones «no hegemónicas».

Estas literaturas son nutridas y documentan las fuerzas interactivas en diferentes partes del mundo que hacen que las zonas fronterizas contemporáneas sean lugares dinámicos. Sin embargo, como señalan académicos como Tony Payan (2014), existen límites significativos en el campo de los estudios de las zonas fronterizas con respecto a la construcción de teorías. Si bien la literatura es variada y prolifera exponencialmente, hay una escasez de estudios comparativos interregionales (Koff, 2007). En consecuencia, la mayor parte de la investigación producida en este campo está restringida por límites regionales. Este punto es especialmente importante con relación al estudio de los impactos de la globalización en las comunidades fronterizas. ¿Cómo pueden determinarse los impactos

\footnotetext{
${ }^{1}$ Este término no tiene aún traducción al español aceptada por la Real Academia Española. Sin embargo, desde su creación por Ole Waever en 1995 se popularizó el concepto en estudios de relaciones internacionales (Treviño, 2016).
} 
de la globalización en estos lugares sin una investigación comparativa interregional, dada la variedad - y a veces superposición- de modelos de integración regional que existen? ¿La globalización impacta en las fronteras europeas de la misma manera que afecta a las fronteras americanas continentales-?

Es interesante notar que gran parte de la literatura en el campo de los estudios sobre las zonas fronterizas indica que la desterritorialización de los mercados, la política y las sociedades provocada por la globalización aumenta la importancia de las zonas fronterizas en los asuntos internacionales. Este punto de vista no es necesariamente compartido en la literatura sobre globalización. Por ejemplo, el número especial La Vie-Le Monde de 2011 documenta una paradoja relacionada con la globalización: cuanto más globalizados se vuelven los sistemas, menos importantes son las comunidades fronterizas, porque las redes transnacionales conectan los centros urbanos sin incluir necesariamente las zonas fronterizas. Esto refuerza los conflictos tradicionales centro-periferia. Además, estudios recientes en el campo de la seguridad describen cómo la regionalización de las estrategias de seguridad beneficia a las capitales nacionales a expensas de las zonas fronterizas (Alès, 2018; Tripathi, 2016).

A través de la exposición de una investigación comparativa, el presente artículo se relaciona con estas literaturas de dos maneras: primero, cuestiona si las comunidades fronterizas tienden a beneficiarse o a sufrir la globalización, y aborda a su vez los impactos de la globalización en las comunidades fronterizas; en segundo lugar, se intenta explicar estos impactos mediante el estudio de la calidad democrática de los procesos de integración fronteriza y se examina así la globalización como un sistema de actores en el que los beneficios se distribuyen o concentran en función de la calidad de los procesos de integración.

\section{Discusión: lecciones de la investigación comparativa sobre globalización y calidad de la democracia en las zonas fronterizas}

Como se dijo anteriormente, el primer objetivo de este artículo es entender los impactos de la globalización en las zonas transfronterizas en diferentes regiones del mundo. Se establece una escala de impacto transfronterizo de la globalización según las reglas explicadas en el apartado 
de la metodología, aunque se presenta como un enfoque metodológico para la comprensión de los impactos de la globalización en las zonas transfronterizas en lugar de una medida absoluta de globalización en los casos de investigación. Los resultados de este análisis se presenten en la tabla 1. Las categorías incluyen la misma cantidad de sectores que representan «beneficios» percibidos y «costos» percibidos de la globalización para no sesgar el estudio. Estas áreas se identifican mediante el examen de indicadores presentes en los índices de globalización internacionales. «Impactos positivos» e «impactos negativos» se refieren a los costos y beneficios de la globalización. Los impactos "generalizados» representan beneficios o costos ampliamente distribuidos, mientras que los impactos concentrados solo afectan a un lado de una comunidad fronteriza (véase el apartado 1).

Tabla 1. Indicadores de globalización para estudios de casos seleccionados.

\begin{tabular}{|c|c|c|c|c|c|c|c|c|}
\hline & Eurometrópolis & $\begin{array}{c}\text { Gran } \\
\text { Región }\end{array}$ & $\begin{array}{l}\text { Melilla- } \\
\text { Nador }\end{array}$ & $\begin{array}{c}\text { Bari- } \\
\text { Durrës }\end{array}$ & $\begin{array}{c}\text { San Diego- } \\
\text { Tijuana }\end{array}$ & $\begin{array}{c}\text { Chetumal- } \\
\text { Corozal }\end{array}$ & $\begin{array}{l}\text { Cúcuta-San } \\
\text { Cristóbal } 1\end{array}$ & $\begin{array}{l}\text { Cúcuta-San } \\
\text { Cristóbal } 2\end{array}$ \\
\hline \multicolumn{9}{|c|}{ Beneficios percibidos } \\
\hline $\begin{array}{l}\text { Cooperación } \\
\text { científica }\end{array}$ & 1 & 1 & 0 & 1 & 1 & 1 & 1 & 1 \\
\hline Turismo & 1 & 0.5 & 0 & 0.5 & 0.5 & 1 & 1 & 1 \\
\hline Comercio & 1 & 1 & 0 & 1 & 1 & 1 & 1 & 0 \\
\hline $\begin{array}{l}\text { Cooperación } \\
\text { de la sociedad } \\
\text { civil }\end{array}$ & 1 & 1 & 0 & 1 & 1 & 0.5 & 1 & 1 \\
\hline $\begin{array}{l}\text { Consumo } \\
\text { transfronterizo }\end{array}$ & 1 & 1 & 0 & 1 & 1 & 1 & 1 & 0 \\
\hline $\begin{array}{l}\text { Flujo de } \\
\text { capital- } \\
\text { inversión }\end{array}$ & 0.5 & 1 & 0 & 1 & 1 & 1 & 1 & 0 \\
\hline $\begin{array}{l}\text { Acceso a } \\
\text { tecnología }\end{array}$ & 1 & 1 & 0 & 1 & 1 & 1 & 0 & 0 \\
\hline $\begin{array}{l}\text { Transporte- } \\
\text { infraestructura }\end{array}$ & 1 & 0 & 0 & 1 & 0 & 0.5 & 0 & 0 \\
\hline $\begin{array}{l}\text { Movilización } \\
\text { transfronteriza }\end{array}$ & 1 & 0,5 & 0 & 1 & 1 & 0 & 1 & 1 \\
\hline $\begin{array}{l}\text { Actividades } \\
\text { culturales }\end{array}$ & 1 & 0.5 & 0 & 0.5 & 1 & 0 & 1 & 0 \\
\hline $\begin{array}{l}\text { Flujos de } \\
\text { información }\end{array}$ & 1 & 1 & 0 & 1 & 1 & 1 & 1 & 0,5 \\
\hline $\begin{array}{l}\text { Intercambios } \\
\text { juveniles }\end{array}$ & 1 & 1 & 0 & 0.5 & 0.5 & 0 & 1 & 0,5 \\
\hline
\end{tabular}


Tabla 1. (Continuación).

\begin{tabular}{|c|c|c|c|c|c|c|c|c|}
\hline & Eurometrópolis & $\begin{array}{l}\text { Gran } \\
\text { Región }\end{array}$ & $\begin{array}{l}\text { Melilla- } \\
\text { Nador }\end{array}$ & \begin{tabular}{|c|} 
Bari- \\
Durrës
\end{tabular} & \begin{tabular}{|c|} 
San Diego- \\
Tijuana
\end{tabular} & \begin{tabular}{|c|} 
Chetumal- \\
Corozal
\end{tabular} & \begin{tabular}{|l|} 
Cúcuta-San \\
Cristóbal 1 \\
\end{tabular} & \begin{tabular}{|l|} 
Cúcuta-San \\
Cristóbal 2 \\
\end{tabular} \\
\hline \multicolumn{9}{|c|}{ Beneficios percibidos } \\
\hline $\begin{array}{l}\text { Programas de } \\
\text { educación }\end{array}$ & 1 & 1 & 0 & 1 & 0 & 0 & 1 & 1 \\
\hline Salud pública & 0 & 1 & 0 & 0.5 & 0.5 & 0 & 0.5 & 0 \\
\hline $\begin{array}{l}\text { Derechos } \\
\text { humanos }\end{array}$ & 0 & 0 & -1 & 0.5 & -1 & -0.5 & 1 & -1 \\
\hline $\begin{array}{l}\text { Derechos de } \\
\text { comunidades } \\
\text { étnicas }\end{array}$ & -1 & 0 & -1 & 1 & -1 & -0.5 & 1 & 0 \\
\hline \multicolumn{9}{|c|}{ Costos percibidos } \\
\hline $\begin{array}{l}\text { Vulnerabilidad } \\
\text { a desastres }\end{array}$ & 0 & 0 & -0.5 & 0 & -0.5 & -1 & -1 & -1 \\
\hline $\begin{array}{l}\text { Presión sobre } \\
\text { recursos } \\
\text { estratégicos }\end{array}$ & -1 & -1 & -1 & -1 & -1 & -1 & -1 & -1 \\
\hline Contaminación & -0.5 & 0 & -0.5 & -1 & -1 & -1 & -1 & -1 \\
\hline $\begin{array}{l}\text { Tráfico- } \\
\text { congestión }\end{array}$ & -1 & -1 & -1 & -1 & -1 & -1 & -0.5 & -1 \\
\hline $\begin{array}{l}\text { Degradación } \\
\text { del suelo }\end{array}$ & -1 & $-0,5$ & -1 & -1 & -0.5 & -0.5 & -0.5 & -1 \\
\hline $\begin{array}{l}\text { Residuos- } \\
\text { disposición }\end{array}$ & $-0,5$ & $-0,5$ & -1 & -1 & -1 & -0.5 & -1 & -1 \\
\hline $\begin{array}{l}\text { Migración no } \\
\text { controlada }\end{array}$ & -1 & 0 & -1 & 1 & -1 & $-0,5$ & 0 & -1 \\
\hline $\begin{array}{l}\text { Tráfico de } \\
\text { personas }\end{array}$ & -1 & 0 & -1 & 1 & -1 & -0.5 & -0.5 & -1 \\
\hline $\begin{array}{l}\text { Tráfico de } \\
\text { drogas }\end{array}$ & -1 & 0 & -1 & -1 & -1 & $-0,5$ & -1 & -1 \\
\hline $\begin{array}{l}\text { Crimen } \\
\text { organizado }\end{array}$ & -1 & 0 & -1 & -1 & -1 & -0.5 & -0.5 & -1 \\
\hline Crimen común & -1 & 0 & -1 & -1 & -1 & -0.5 & -0.5 & -1 \\
\hline Informalidad & -1 & $-0,5$ & -1 & -1 & -1 & -0.5 & -1 & -1 \\
\hline $\begin{array}{l}\text { Explotación } \\
\text { laboral }\end{array}$ & -0.5 & -1 & 0 & -0.5 & -1 & -1 & -0.5 & -0.5 \\
\hline $\begin{array}{l}\text { Déficit } \\
\text { habitacional }\end{array}$ & -0.5 & -1 & -0.5 & -0.5 & -1 & 0 & -1 & -1 \\
\hline $\begin{array}{l}\text { Inequidad } \\
\text { social }\end{array}$ & -1 & -1 & -1 & -0.5 & -1 & -1 & 0 & -1 \\
\hline Pobreza & -1 & 0 & -1 & 0.5 & -1 & -0.5 & 0 & -1 \\
\hline Total & -1.5 & +3.5 & -15.5 & +5 & -6.5 & -4.5 & +3.5 & -10.5 \\
\hline
\end{tabular}

Fuente: elaboración propia. 
La tabla 1 presenta consideraciones significativas relacionadas con la comprensión de la globalización y las zonas transfronterizas. Primero, debido a que la mayoría de los puntajes generales son negativos, indica que las zonas fronterizas están expuestas a la globalización sin necesariamente recibir beneficios. Solo la Gran Región de Luxemburgo, Bari-Durrës y Cúcuta-San Cristóbal, antes de que Venezuela se retirara de la Comunidad Andina, parecen beneficiarse de la globalización sin asumir costos desorbitados.

El segundo hallazgo que se deriva de estos datos es que las agrupaciones presentes en el cuadro 1 no parecen existir. La mayoría de los casos europeos demuestran puntajes menos costosos que los estadounidenses y la salida de Venezuela de la CAN parece impactar negativamente a Cúcuta y San Cristóbal. Sin embargo, existe una variación significativa entre los casos que no refleja las agrupaciones que representan los paradigmas de la globalización. Las agrupaciones regionales no se reflejan en la tabla 2. Resultados inesperados muestran que Bari-Durrës se beneficia de la globalización más que la Eurometrópolis, a pesar de la posición de esta última como una metrópolis transfronteriza patrocinada por la UE. Además, la Eurometrópolis se caracteriza por los costes de la globalización que se acercan más al caso Chetumal-Corozal que a la Gran Región de Luxemburgo, el otro caso interno de la UE. Si bien estos casos son muy diferentes, ambos demuestran concentraciones de los beneficios de la globalización combinados con la marginación social transfronteriza que genera costos significativos.

Asimismo, tampoco emergen agrupaciones ideológicas y de movilización. Bari-Durrës presenta los mayores beneficios generales de la globalización, seguida por la Gran Región de Luxemburgo y Cúcuta-San Cristóbal 1. Melilla-Nador se ha caracterizado por los costos generales más altos, seguida de Cúcuta-San Cristóbal 2. Estos casos son aquellos en los que la globalización se limita a beneficios concentrados con costos generalizados. Estos resultados sugieren que las tres principales explicaciones conceptuales de los impactos de la globalización no explican adecuadamente la interacción entre globalización y desarrollo en las zonas transfronterizas. En consecuencia, este artículo propone un análisis basado en la «calidad de la democracia». 


\subsection{Calidad de la democracia y los impactos de la globalización en las zonas transfronterizas}

Diversos estudios sobre integración fronteriza identifican claramente la necesidad de la participación ciudadana en los procesos de toma de decisiones. De un lado, Payan (2016) sostiene que el déficit democrático en las políticas de seguridad fronteriza contribuye a estrategias con efectos adversos en las ciudades fronterizas de América del Norte. De manera similar, Carmen Maganda $(2013 ; 2008)$ argumenta que la regionalización de las políticas ambientales transfronterizas en Europa y Sur América disminuye la cantidad de información pública sobre temas ambientales, lo que afecta negativamente la capacidad de las comunidades fronterizas para tomar decisiones informadas. Por el contrario, Stephen Mumme, Oscar Ibáñez y Suzanne Till, (2012) construyen un modelo de gobernanza multinivel basado en la participación ciudadana en la gestión del agua en la frontera México-Estados Unidos. Del mismo modo, Pamela Cruz (2014) destaca la necesidad de participación en la implementación de políticas transfronterizas y John Agnew (2020) y Nadalutti (2020) demuestran cómo los proyectos transfronterizos de la Unión Europea son efectivos si la participación de las partes interesadas está integrada.

Estos trabajos documentan casos en los que la participación democrática es necesaria para la formulación de políticas eficaces en las zonas transfronterizas; sin embargo, la construcción de teorías es limitada porque existen estudios similares en los que la participación social en la integración transfronteriza tiene impactos negativos. Académicos como Marcela Ceballos (2011) y Harlan Koff (2015) demuestran cómo la movilización transfronteriza en realidad contribuye al conflicto social, especialmente en estados frágiles ubicados en Asia, África y Sur América. Estos estudios indican que la actividad de la sociedad civil permite a los gobiernos locales abandonar sus responsabilidades con los ciudadanos fronterizos o, lo que es más problemático, establece autoridades paralelas que desafían la legitimidad de los gobiernos y debilitan aún más a los Estadosnación. Por su parte, Cúcuta-San Cristóbal (Koff, 2018) es crucial para este estudio porque representa un caso en el que existe una larga tradición de participación social en la política transfronteriza, pero esta movilización transnacional no afecta positivamente la formulación de políticas formales o el desarrollo. Este artículo examina este rompecabezas. 
Si la participación social no es suficiente para afectar positivamente la integración fronteriza, ¿qué marco puede explicar los niveles de globalización y los impactos sociales? En respuesta, este artículo importa el paradigma de la "calidad de la democracia» (Diamond y Morlino, 2004) al campo de los estudios de las zonas fronterizas. La importancia del enfoque de la calidad de la democracia es su capacidad para integrar consideraciones éticas y morales en un marco analítico que es relativamente objetivo. Larry Diamond y Leonardo Morlino (2004), de hecho, señalan ocho indicadores que identifican como piedras angulares de las democracias de «calidad»: Estado de derecho, participación, competencia, rendición de cuentas vertical, rendición de cuentas horizontal, libertad, igualdad y capacidad de respuesta.

Este enfoque responde a dos necesidades para la construcción de teorías: primero, se puede aplicar a diferentes contextos geográficos y políticos. La investigación sobre el tema se ha centrado en la implementación del marco de Diamond y Morlino en regiones y países específicos - Sebastián Mazzuca (2010) para América Latina; Jan Zielonka (2007) para Europa; otras obras intentan operacionalizar este enfoque a través de datos empíricos comparativos (Campbell, 2008)—. La base de datos comúnmente citada construida alrededor de este paradigma es el Índice de Transformación de Bertelsmann (BTI, s. f.), que proporciona medidas cuantitativas sobre estos ocho indicadores para países de todo el mundo.

En segundo lugar, este enfoque para el estudio de la democracia proporciona un marco analítico que se centra en los mecanismos de toma de decisiones en los sistemas democráticos. De este modo, la participación social es solo un indicador bajo este enfoque. Al examinar los mecanismos, este paradigma analiza tanto las características de las democracias de «buena calidad» como las relaciones entre estas características. En consecuencia, este enfoque ayuda a comprender por qué la participación social es efectiva en algunos contextos transfronterizos, pero ineficaz e incluso peligrosa en otros. La participación social suele filtrarse a través de organismos intermediarios como partidos políticos, movimientos sociales y ONG. Así, el enfoque de la calidad de la democracia da cuenta de las estructuras formales e informales en los sistemas democráticos. 


\subsection{Calidad de la democracia y la globalización en las zonas transfronterizas: consideraciones empíricas}

Dado que no existen datos de calidad de la democracia transfronteriza, este artículo se basa en escalas derivadas de la investigación cualitativa sobre las ocho dimensiones identificadas por Diamond y Morlino (2004). la libertad se refiere a las libertades tanto formales como sustantivas de las que disfrutan los ciudadanos para expresar opiniones y movilizarse políticamente; el Estado de derecho incluye características como la presencia de un poder judicial imparcial, la falta de corrupción y transparencia, las cuales impactan la confianza de los residentes en las instituciones y funcionarios públicos; la rendición de cuentas vertical se define como la supervisión pública a los funcionarios gubernamentales y la rendición de cuentas horizontal se refiere a las relaciones dentro del gobierno a través de las cuales los funcionarios públicos se responsabilizan entre sí; la capacidad de respuesta se refiere a la eficacia con la que los creadores de las políticas responden a las necesidades de los residentes; la igualdad incluye consideraciones formales y sustantivas relacionadas con la distribución equitativa de derechos y responsabilidades; la participación se refiere al aporte público en los procesos de formulación de políticas; y la competitividad se refiere a la presencia de una oposición democrática viable.

Tabla 2. Indicadores de calidad de la democracia para casos de investigación seleccionados.

\begin{tabular}{|l|c|c|c|c|c|c|c|c|}
\hline & Eurometrópolis & $\begin{array}{c}\text { Gran } \\
\text { Región }\end{array}$ & $\begin{array}{c}\text { Melilla- } \\
\text { Nador }\end{array}$ & $\begin{array}{c}\text { Bari- } \\
\text { Durrës }\end{array}$ & $\begin{array}{c}\text { San } \\
\text { Diego- } \\
\text { Tijuana }\end{array}$ & $\begin{array}{c}\text { Chetumal- } \\
\text { Corozal }\end{array}$ & $\begin{array}{c}\text { Cúcuta- } \\
\text { San } \\
\text { Cristóbal } \\
\mathbf{1}\end{array}$ & $\begin{array}{c}\text { Cúcuta- } \\
\text { San } \\
\text { Cristóbal } \\
\mathbf{2}\end{array}$ \\
\hline Libertad & 0.5 & 1 & 0.5 & 0.5 & 0.5 & 0.5 & 0 & -0.5 \\
\hline $\begin{array}{l}\text { Estado de } \\
\text { derecho }\end{array}$ & 0.5 & 0.5 & -0.5 & 0.5 & -0.5 & 0 & -0.5 & -1 \\
\hline $\begin{array}{l}\text { Rendición } \\
\text { de cuentas } \\
\text { vertical }\end{array}$ & 0.5 & -0.5 & -1 & 0.5 & -1 & -1 & 0,5 & -1 \\
\hline $\begin{array}{l}\text { Rendición } \\
\text { de cuentas } \\
\text { horizontal }\end{array}$ & 0.5 & 0.5 & -1 & 0.5 & -0.5 & -1 & 0.5 & -1 \\
\hline
\end{tabular}


Tabla 2. (Continuación).

\begin{tabular}{|l|c|c|c|c|c|c|c|c|}
\hline & Eurometrópolis & $\begin{array}{c}\text { Gran } \\
\text { Región }\end{array}$ & $\begin{array}{c}\text { Melilla- } \\
\text { Nador }\end{array}$ & $\begin{array}{c}\text { Bari- } \\
\text { Durrës }\end{array}$ & $\begin{array}{c}\text { San } \\
\text { Diego- } \\
\text { Tijuana }\end{array}$ & $\begin{array}{c}\text { Chetumal- } \\
\text { Corozal }\end{array}$ & $\begin{array}{c}\text { Cúcuta- } \\
\text { San } \\
\text { Cristóbal } \\
\mathbf{1}\end{array}$ & $\begin{array}{c}\text { Cúcuta- } \\
\text { San } \\
\text { Cristóbal } \\
\mathbf{2}\end{array}$ \\
\hline $\begin{array}{l}\text { Capacidad de } \\
\text { respuesta }\end{array}$ & 0.5 & 1 & -1 & 1 & -1 & -0.5 & -0.5 & -1 \\
\hline Igualdad & -1 & 0.5 & -1 & -1 & -1 & -0.5 & 0 & -0.5 \\
\hline Participación & -0.5 & 0 & -0.5 & -0.5 & 0 & -0.5 & 1 & 0.5 \\
\hline Competición & -1 & -1 & -1 & 0.5 & -1 & -0.5 & -1 & -1 \\
\hline Total & 0 & 2 & -5 & 2 & $-4,5$ & $-3,5$ & 0 & -5.5 \\
\hline
\end{tabular}

Fuente: elaboración propia.

El segundo objetivo de este artículo es comprender qué tan bien la calidad de la democracia transfronteriza explica los impactos de la globalización en las zonas transfronterizas. La gráfica 1 compara estos puntajes de "calidad de la democracia» con los puntajes de globalización presentados en la tabla 1. La gráfica 1 indica una estrecha relación entre la calidad de la democracia en los casos investigados y la interacción de la globalización con estos mismos casos.

Gráfica 1. Relación entre la calidad de la democracia y la globalización en los casos seleccionados.

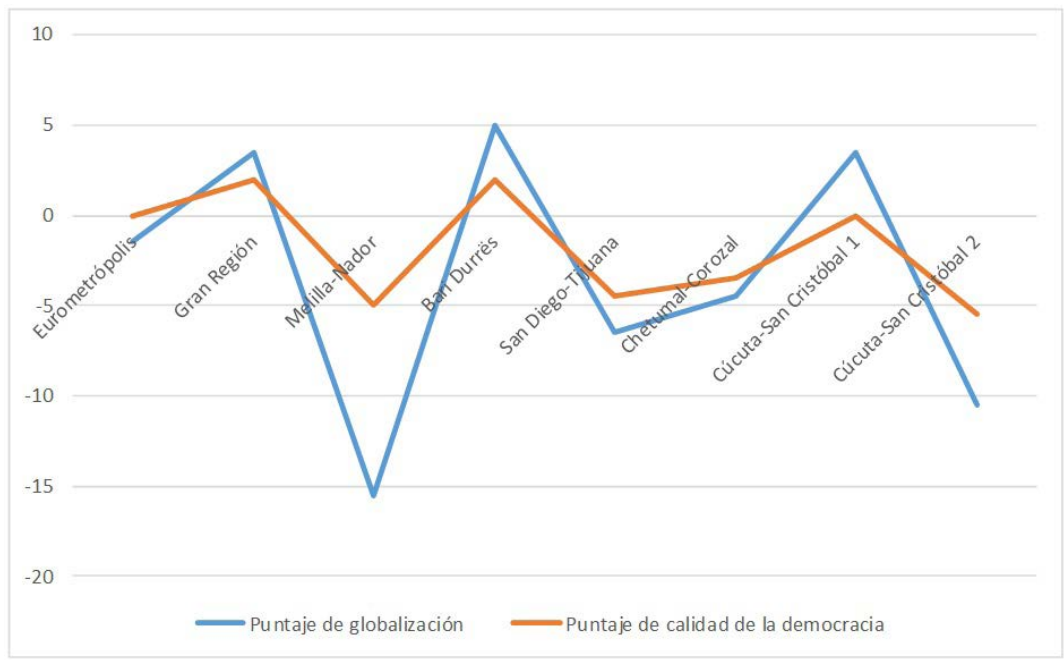

Fuente: elaboración propia. 
Estas tendencias son significativas ya que explican por qué los puntajes de globalización son negativos en casos caracterizados por altos niveles de participación social, como Cúcuta-San Cristóbal. La participación es, de hecho, solo una característica de una democracia de buena calidad. En la Gran Región de Luxemburgo, Bari-Durrës y Cúcuta-San Cristóbal 1, en los casos que mostraron puntajes de globalización positivos, los modelos de integración fronteriza se establecen a través de discusiones democráticas amplias. En el caso de la Gran Región de Luxemburgo los funcionarios gubernamentales promueven la integración a través de estructuras de gobernanza corporativa que integran a actores económicos y políticos de diferentes áreas de la Gran Región. Esto explica la eficacia de la planificación económica, así como la concentración de beneficios en Luxemburgo a expensas de las áreas fronterizas circundantes, dado que los actores luxemburgueses representan intereses nacionales, mientras que los participantes de Bélgica, Francia y Alemania representan zonas que están marginadas en sus propios regímenes nacionales.

En Bari-Durrës los beneficios de la integración son más difusos porque los actores locales promueven procesos de integración de abajo hacia arriba desde la década de 1990, momento en que el liderazgo de Bari fue diezmado por el escándalo de corrupción Tangentopoli y la caída del comunismo en Durrës. Ambos países también padecieron para la época problemas estructurales similares, incluidos el desempleo crónico, la corrupción y el crimen organizado. Por estas razones se iniciaron los procesos de integración fronteriza entre miembros de la sociedad civil y las comunidades empresariales locales. Una vez que se establecieron estas interacciones, las autoridades locales, nacionales y de la UE apoyaron esta cooperación con inversiones e infraestructura. A pesar de que se trata de una frontera exterior de la UE, la globalización tiene un impacto positivo tanto en Bari como en Durrës porque la integración fronteriza en esta región siguió un modelo democrático.

De manera similar, la integración regional impacta positivamente la integración fronteriza en Cúcuta y San Cristóbal mientras Venezuela fue parte de la Comunidad Andina. Durante ese periodo la integración ocurrió a través de Zonas de Integración Fronteriza (ZIF), formalmente reconocidas e institucionalizadas por la Comunidad Andina, la cual asegura la participación de los miembros de las comunidades transfronterizas, la colaboración de la sociedad civil y la cooperación del gobierno local. 
Esto impacta positivamente en la rendición de cuentas y en la supervisión institucional tanto regional como de gobiernos nacionales, lo cual mejora el Estado de derecho.

Estos casos «exitosos» indican que las estrategias locales de desarrollo transfronterizo tienen en cuenta la globalización como un proceso, como una ideología económica y un sistema impulsado por actores, ya que aprovechan las estructuras de oportunidad regionalizadas, presentan modelos transparentes de gobernanza económica y proporcionan diferentes puntos de acceso para la pluralidad en la toma de decisiones. En resumen, el desarrollo transfronterizo de «buena calidad» se acompaña de procesos democráticos transfronterizos de «buena calidad» que dan cuenta de las tres visiones de la globalización.

Por el contrario, los tres «puntajes de globalización» más bajos se encuentran en Melilla-Nador, San Diego-Tijuana y Cúcuta-San Cristóbal 2, las fronteras más militarizadas del estudio. Melilla-Nador y San DiegoTijuana son casos divididos por muros fronterizos. Cúcuta-San Cristóbal 2 incluye una frontera que las autoridades venezolanas y colombianas cierran periódicamente por preocupaciones de seguridad relacionadas con la extensión del conflicto armado de Colombia, disputas de peaje en la carretera Simón Bolívar y la agitación política en Venezuela. La presencia de una gobernanza fronteriza militarizada no solo divide a las poblaciones e impide la movilización social, sino que, sobre todo, afecta la transparencia, al Estado de derecho y a la rendición de cuentas en las zonas transfronterizas. Además, el aumento del crimen organizado en respuesta a esta militarización afecta negativamente la libertad en las comunidades fronterizas. Esto explica por qué estas áreas «sufren» la globalización en términos de costos sociales, a pesar de que algunas de ellas, como San Diego-Tijuana, se benefician enormemente en términos de riqueza general.

\section{Conclusión}

Este artículo propone dos preguntas de investigación: ¿cómo ha afectado la globalización a las zonas fronterizas? y ¿qué explica estos impactos? En el apartado anterior se utilizaron escalas para medir la globalización y sus impactos en las zonas fronterizas. Estas escalas armonizan los datos cualitativos en las diferentes regiones del mundo estudiadas. Dichas escalas indican que las zonas fronterizas están expuestas 
a los costos de la globalización, sin necesariamente recibir sus beneficios. Además, los casos de investigación demuestran claras tendencias en relación con la globalización. Por un lado, algunas de estas zonas transfronterizas se integran en economías globalizadas y aumentan las conexiones transfronterizas. En otros casos, procesos como la securitización de fronteras obstaculizan las conexiones transfronterizas. De manera similar, ciertos casos de investigación se caracterizan por costos y beneficios compartidos de la globalización, mientras que estos costos y beneficios se concentran en otros casos. Estas tendencias se ilustran en el cuadro 2.

Cuadro 2. Tipologías de globalización en zonas transfronterizas: resultados empíricos.

\begin{tabular}{|c|c|c|}
\hline & $\begin{array}{c}\text { Aumento general de las } \\
\text { interconexiones transfronterizas }\end{array}$ & $\begin{array}{c}\text { Aumento limitado de las } \\
\text { interconexiones transfronterizas }\end{array}$ \\
\hline $\begin{array}{l}\text { Amplia distribución de } \\
\text { costos y beneficios }\end{array}$ & $\begin{array}{l}\text { - Bari-Durrës. } \\
\text { - Gran Región de Luxemburgo. }\end{array}$ & - Cúcuta-San Cristóbal 1. \\
\hline $\begin{array}{l}\text { Concentraciones de } \\
\text { costos y beneficios }\end{array}$ & $\begin{array}{l}\text { - San Diego-Tijuana. } \\
\text { - Eurometrópolis. } \\
\text { - Chetumal-Corozal. }\end{array}$ & $\begin{array}{l}\text { - Melilla-Nador. } \\
\text { - Cúcuta-San Cristóbal } 2 .\end{array}$ \\
\hline
\end{tabular}

[316] Fuente: elaboración propia.

Esta investigación interregional y las tendencias que emergen entre los casos empíricos abren vías interesantes en términos de teorías de integración fronteriza y de la globalización. Primero, ofrecen la oportunidad de formular tipologías de globalización transfronteriza basadas en la interacción entre las interconexiones transfronterizas y la distribución de costos y beneficios. Estas tipologías se presentan en el cuadro 3.

Cuadro 3. Tipologías de globalización en zonas transfronterizas.

\begin{tabular}{|l|l|l|}
\hline & \multicolumn{1}{|c|}{$\begin{array}{c}\text { Aumento general de las } \\
\text { interconexiones transfronterizas }\end{array}$} & $\begin{array}{c}\text { Aumento limitado de las } \\
\text { interconexiones transfronterizas }\end{array}$ \\
\hline $\begin{array}{l}\text { Amplia distribución de } \\
\text { costos y beneficios }\end{array}$ & Globalización compartida & Globalización bloqueada \\
\hline $\begin{array}{l}\text { Concentración de } \\
\text { costos y beneficios }\end{array}$ & Globalización desigual & Globalización marginalizada \\
\hline
\end{tabular}

Fuente: elaboración propia. 
En relación con la segunda pregunta planteada: ¿qué explica los impactos de la globalización en las regiones fronterizas?, es importante señalar que aquellos casos donde los impactos de la globalización se han caracterizado por mayores interconexiones y costos y beneficios compartidos —el mejor escenario»- son aquellos con los niveles más altos de la calidad de democracia transfronteriza. Al contrario, los casos caracterizados por interconexiones limitadas y costos y beneficios concentrados - «el peor escenario»- se caracterizan por el más bajo puntaje de calidad de la democracia transfronteriza.

Este enfoque destaca la importancia de profundizar las discusiones actuales sobre democracia en las zonas fronterizas más allá de la participación social. Sobre todo, ilustra la necesidad de aplicar las herramientas que se usan para evaluar la democratización en la integración transfronteriza. Estos procesos deben ser participativos, receptivos y transparentes para que las zonas fronterizas generen y se beneficien de las interconexiones transnacionales. Si los ciudadanos participan en los procesos de globalización en asociación con las instituciones, se puede lograr una globalización compartida que se caracteriza por mayores interconexiones y beneficios generalizados. Por el contrario, mientras se excluye a los ciudadanos de estos procesos — globalización desigual-, participan en ellos sin coordinarse con las autoridades gubernamentales globalización marginalizada-, las comunidades fronterizas sufren mayores costos y menores beneficios de la globalización, lo que actualmente parece ser la norma en muchas partes del mundo.

\section{Referencias bibliográficas}

1. Agnew, John. (2020). Revisiting Europe in Search of Regional Cohesion. Regions \& Cohesion, 10 (3), pp. 1-9. https://doi.org/10.3167/reco.2020.100303

2. Alès, Catherine. (2018). From Proclamation to Denial: Indigenous Rights and Political Participation in Venezuela. Regions \& Cohesion, 8 (2), pp. 49-81. https://doi. org/10.3167/reco.2018.080204

3. Barber, Benjamin. (1995). Jihad vs. Mc World. New York: Ballantine.

4. Brightman, Marc \& Grotti, Vanessa. (2014). Securitization, Alterity, and the State. Human (In)Security on an Amazonian Frontier. Regions \& Cohesion, 4 (3), pp. 17-38. https://doi.org/10.3167/reco.2014.040302

5. BTI Transformation Index. (s. f.). The Transformation Index. https://bti-project. org/en/?\&cb $=00000$ 
6. Campbell, David. (2008). The Basic Concept of the Democracy Ranking for the Quality of Democracy. Vienna: Democracy Ranking.

7. Castells, Manuel. (2003). The Internet Galaxy. Oxford: Oxford University.

8. Ceballos Medina, Marcela. (2011). La política migratoria de Ecuador hacia Colombia. Entre la integración y la «contención». Regions \& Cohesion, 1 (2), pp. 4577. https://doi.org/10.3167/reco.2011.010204

9. Cruz, Pamela. (2014). Cross-Border Governance on the U.S.-Mexico Border: Institutional Challenges and Developments in Health Collaboration. Regions \& Cohesion, 4 (1), pp. 53-71. https://doi.org/10.3167/reco.2014.040104

10. Choo Chin, Low. (2019). Extraterritorial Migration Control in Malaysia. Militarized, Externalized, and Regionalized. Regions \& Cohesion, 9 (3), pp. 1-28. https://doi.org/10.3167/reco.2019.090302

11. De la Mora-De la Mora, Gabriela. (2017). Policentrismo y su relevancia para el análisis socioterritorial. Características, enfoques y dimensiones analíticas. Regions \& Cohesion, 7 (1), pp. 69-86. https://doi.org/10.3167/reco.2017.070106

12. Denis, Jean-Pierre \& Greilsamer, Laurent (Eds.). (2010). L'Atlas des Mondialisations. Paris: La Vie-Le Monde.

13. Diamond, Larry \& Morlino, Leonardo. (2004). The Quality of Democracy: An Overview. Journal of Democracy, 15 (4), pp. 20-31. https://doi.org/10.1353/ jod.2004.0060

14. Dreher, Axel \& Gaston, Noel. (2008). Has Globalisation Increased Inequality?

[318] Review of International Economics, 16 (3), pp. 516-536. https://doi.org/10.1111/ j.1467-9396.2008.00743.x

15. Dreher, Axel; Gassebner, Martin \& Siemers, Lars. (2012). Globalisation, Economic Freedom and Human Rights. The Journal of Conflict Resolution, 56 (3), pp. 516-546. https://doi.org/10.1177/0022002711420962

16. Dubé, Sébastien. (2020). A Supposed Fifth Wave of Latin American Regionalism and Human Needs. Regions \& Cohesion, 10 (3), pp. 38-71. https://doi. org/10.3167/reco.2020.100307

17. Elster, Jon. (1992). Local Justice. New York: Russell Sage Foundation.

18. ETH Zürich KOF. (s. f.). KOF Globalisation Index. https://kof.ethz.ch/en/ forecasts-and-indicators/indicators/kof-globalisation-index.html

19. Faleye, Olukayode. (2016). Regional Integration from «Below» in West Africa: A Study of Transboundary Town-Twinning of Idiroko (Nigeria) and Igolo (Benin). Regions \& Cohesion, 6 (3), pp. 1-19. https://doi.org/10.3167/reco.2016.060301

20. Fligstein, Niel. \& Habinek, Jacob. (2014). Sucker-punched by the Invisible Hand: The World Financial Markets and the Globalisation of the US Financial Crisis. Socio-Economic Review 12, pp. 1-29. https://doi.org/10.1093/ser/mwu004

21. Gerber, James. (2020). Governed and Ungoverned Integration in the MexicoUS Border Region. Regions \& Cohesion, 10 (2), pp. 21-40. https://doi.org/10.3167/ reco.2020.100203 
22. Gereffi, Gary. (1994). The Organization of Buyer-driven Global Commodity Chains. In: Gereffi, Gary \& Korzeniewicz, Miguel (Eds.). Commodity Chains and Global Capitalism (pp. 95-122). Westwood: Greenwood.

23. Giddens, Anthony. (2000). Runaway World: How Globalisation is Reshaping our Lives. New York: Routledge.

24. Guillén, Mauro F. (2001). Is Globalisation Civilizing, Destructive or Feeble? A Critique of Five Key Debates in the Social Science Literature. Annual Review of Sociology, 27, pp. 235-260. https://doi.org/10.1146/annurev.soc.27.1.235

25. Häbel, Sandra. (2020). Normative policy coherence for development and policy networks: EU networks in Vietnam. Regions \& Cohesion, 10 (1), pp. 1-21. https://doi.org/10.3167/reco.2020.100102

26. Held, David; McGrew, Anthony; Goldblatt, David \& Perraton, Jonathan (Eds.). (1999). Global Transformations. Palo Alto: Stanford University.

27. Herzog, Lawrence \& Sohn, Christophe. (2014). The Cross-Border Metropolis in a Global Age: A Conceptual Model and Empirical Evidence from the US-Mexico and European Border Regions. Global Society, 28 (4), pp. 441-461. https://doi.org/10 $.1080 / 13600826.2014 .948539$

28. Hu, Zhiding y Konrad, Victor. (2021). Repositioning Security Spaces of Exclusion, Exception, and Integration in China-Southeast Asia Borderlands. Regions \& Cohesion, 11 (2), pp. 1-25. https://doi.org/10.3167/reco.2021.110202

29. Kay, Sean. (2004). Globalisation, Power and Security. Security Dialogue, 35 (1), pp. 9-25. https://doi.org/10.1177/0967010604042533

30, Koff, Harlan (Ed.). (2007). Deceiving (Dis)Appearances: Analyzing Current Developments in European and North American Border Regions. Brussels: P.I.E.Peter Lang.

31. Koff, Harlan. (2015). Informal Economies in European and American CrossBorder Regions: A Comparative Framework. Journal of Borderlands Studies, 30 (4), pp. 469-487. https://doi.org/10.1080/08865655.2016.1165133

32. Koff, Harlan. (2017). Policy Coherence for Development and Migration: Analyzing US and EU Policies Through the Lens of Normative Transformation. Regions and Cohesion, 7 (2), pp. 5-33. https://doi.org/10.3167/reco.2017.070202

33. Koff, Harlan. (2018) Outside-in Region-building: The Role of Border Integration Zones in Andean Regional Integration. In: Nadalutti, Elisabetta \& Kallscheuer, Otto (Eds.). Region-making and Cross-border Cooperation: New Evidence from Four Continents (pp. 62-80). London: Routledge. https://doi.org/10.9774/ gleaf.9781315195605_6

34. Liberona, Nanette; Álvarez, Camila y Córdoba, Gabriela. (2018). Procesos de fronterización y desfronterización en territorios latinoamericanos. Polis, 51, pp. 1-6. https://doi.org/10.32735/S0718-6568/2018-N51-1361

35. Lins Ribeiro, Gustavo. (2009). Non-hegemonic Globalisations. Anthropological Theory, 9 (3), pp. 297-329. https://doi.org/10.1177/1463499609346985 
36. Maganda, Carmen. (2008). ¿Agua dividida, agua compartida? Acuíferos transfronterizos en sudamérica, una aproximación. Estudios Politicos, 32, pp. 171-194.

37. Maganda, Carmen. (2013). The Implementation of the European Water Framework Directive in Luxembourg: Regional Compliance vs. Cross-Border Cooperation. International Journal of Water Governance, 1 (3), pp. 403-426. https:// doi.org/10.7564/13-IJWG15

38. Martens, Pim \& Raza, Mohsin. (2010). Is Globalisation Sustainable?. Sustainability, 2, pp. 280-293. https://doi.org/10.3390/su2010280

39. Mazzuca, Sebastián. (2010). Access to Power Versus Exercise of Power: Reconceptualizing the Quality of Democracy in Latin America. Studies in Comparative International Development, 45, pp. 334-357. https://doi.org/10.1007/s12116-0109069-5

40. Meissner, Richard \& Warner, Jeroen. (2021). Indigenous Paradiplomacy and the Orokawe Hydroelectric Dam on the Kunene River. Regions \& Cohesion, 11 (2), pp. 21-48. https://doi.org/10.3167/reco.2021.110103

41. Morales, Diana. (2020). Conceptualizing Sub-national Regional Cooperation. Coffee Cultural Landscape of Colombia Case Study. Regions \& Cohesion, 10 (1), pp. 61-87. https://doi.org/10.3167/reco.2020.100105

42. Mumme, Stephen; Ibáñez, Oscar \& Till, Suzanne. (2012) Multilevel Governance of Water on the U.S.-Mexico Border. Regions \& Cohesion, 2 (2), pp. 6-29. https://doi.org/10.3167/reco.2012.020202

[320] 43. Nadalutti, Elisabetha. (2020). The Ethics of Cross-border Cooperation and its Values. Regions \& Cohesion, 10 (2), pp. 42-63. https://doi.org/10.3167/ reco.2020.100204

44. Nhengu, Dudziro. (2020). The Health of Migrants as a Global Peace and Security Agenda. Regions \& Cohesion, 10 (2), pp. 1-20. https://doi.org/10.3167/ reco.2020.100202

45. Ohmae, Kenichi. (1999). The Borderless World. New York: Harper Business.

46. Payan Tony. (2016). The Three U.S.-Mexico Border Wars: Drugs, Immigration, and Homeland Security. Santa Barbara: Praeger.

47. Payan, Tony. (2014). Theory-building in Border Studies: The View from North America. Eurasia Border Review, 5 (1), pp. 1-18.

48. Pim Martens.info. (s. f.). Maastricht Globalization Index. http://pimmartens. info/research/globalisation-index/

49. Rogowski, Ronald. (1990). Commerce and Coalitions. Princeton: Princeton University. https://doi.org/10.1515/9780691219431

50. Rossi, Ino. (Ed.) (2007). Frontiers of Globalisation Research. New York: Springer. https://doi.org/10.1007/978-0-387-33596-4

51. Sandoval Palacios, Juan Manuel. (2017). US-México Border States and the US Military-Industrial Complex. A Global Space for Expanding Transnational Capital. Regions \& Cohesion, 7 (1), pp. 87-121. https://doi.org/10.3167/reco.2017.070107 
52. Sassen, Saskia. (2010). L'émergence d'une nouvelle géographie transnationale. In: Denis, Jean-Pierre \& Greilsamer, Laurent (Eds.). L'Atlas des Mondialisations (pp. 10-12). Paris: La Vie-Le Monde.

53. Scholte, Jan Aart. (2004). Globalisation Studies: Past and Future: A Dialogue of Diversity. Globalisations 1 (1), pp. 102-110. https://doi. org/10.1080/1474773042000252183

54. Tainio, Risto; Meriläinen, Susan; Mäkinen, Jukka \& Laihonen, Maarit (Eds.). (2014). Limits to Globalisation. Copenhagen: Copenhagen Business School.

55. Treviño Rangel, Javier. (2016). ¿De qué hablamos cuando hablamos de la «securitización» de la migración internacional en México?: una crítica. Foro Internacional, 56 (2), pp. 253-291.

56. Tripathi, Dhananjay. (2016). Creating Borders in Young Minds: A Case Study of Indian and Pakistani School Textbooks. Regions \& Cohesion, 6 (1), pp. 52-71. https://doi.org/10.3167/reco.2016.060103

57. Vivekanandan, Jayashree. (2021). Scratches on Our Sovereignty?: Analyzing Conservation Politics in the Sundarbans. Regions \& Cohesion, 11 (1), pp. 1-20. https:// doi.org/10.3167/reco.2021.110102

58. Zembylas, Michalinos \& Vrasidas, Charalambos. (2005). Globalisation, Information and Communication Technologies, and the Prospect of a "Global Village»: Promises of Inclusion or Electronic Colonization?. Journal of Curriculum Studies, 37 (1), pp. 65-83. https://doi.org/10.1080/0022027032000190687

59. Zielonka, Jan. (2007). The Quality of Democracy After Joining the European Union. East European Politics and Societies, 21 (1), pp. 162-180. https://doi. org/10.1177/0888325406297133 